\title{
Attributional styles and stress-related atherogenic plasma lipid reactivity in essential hypertension
}

\author{
Ulrike Kuebler $^{\text {a }}$, Manuel Trachsel ${ }^{\text {b }}$, Roland von Känel ${ }^{\text {c,d }}$, Elvira Abbruzzese ${ }^{\text {a }}$, Ulrike Ehlert ${ }^{\mathrm{a}}$, Petra H. Wirtz ${ }^{\mathrm{e}, *}$ \\ a Clinical Psychology and Psychotherapy, University of Zurich, Zurich, Switzerland \\ ${ }^{\mathrm{b}}$ Institute of Biomedical Ethics, University of Zurich, Zurich, Switzerland \\ c Division of Psychosomatic Medicine, Department of General Internal Medicine, Inselspital, Bern University Hospital, and University of Bern, Bern, Switzerland \\ d Department of Clinical Research, University of Bern, Bern, Switzerland \\ e Biological and Health Psychology, University of Bern, Bern, Switzerland
}

\section{Keywords:}

Attributional styles

Cholesterol

Low-density-lipoprotein cholesterol

Hypertension

Plasma lipids

Psychosocial stress

Trier Social Stress Test

\begin{abstract}
A B S T R A C T
Objective: Hypertension and an atherogenic lipid profile are known risk factors for coronary heart disease (CHD). Hypertensives show greater changes in atherogenic plasma lipids to acute stress than normotensives. In this study, we investigated whether attribution of failure is associated with lipid stress reactivity in hypertensive compared with normotensive men.

Methods: 18 normotensive and 17 hypertensive men (mean \pm SEM; $45 \pm 2.2$ years) underwent an acute stan dardized psychosocial stress task that can be viewed as a situation of experimentally induced failure. We assessed external stable (ES), external variable (EV), internal stable (IS), and internal variable (IV) attribution of failure and psychological control variables (i.e. extent of depression and neuroticism). Moreover, total cholesterol (TC), low density lipoprotein cholesterol (LDL C), and norepinephrine were measured immediately before and several times after stress.

Results: ES moderated TC and LDL C stress reactivity in hypertensives as compared to normotensives (interaction mean arterial pressure [MAP] by ES for TC: $F=3.71, p=.015$; for LDL C: $F=3.61, p=.016)$. TC and LDL C levels were highest in hypertensives with low ES immediately after stress $(p \leq .039)$. In contrast, hypertensives with high ES did not differ from normotensives in TC and LDL C immediately after stress ( $p$ 's $>.28$ ). Controlling for norepi nephrine, depression, and neuroticism in addition to age and BMI did not significantly change results. There were no significant associations between lipid baseline levels or aggregated lipid secretion and IS, IV, or EV ( $p$ 's $>.23$ ). Conclusion: Our data suggest that ES may independently protect from elevated lipid stress reactivity in hypertensive individuals. ES thus might be a protective factor against CHD in hypertension.
\end{abstract}

\section{Introduction}

Essential hypertension ranks among the main risk factors for coro nary heart disease (CHD) [1] but the underlying mechanisms are not fully understood. A well known classic risk factor for CHD is an athero genic lipid profile with increased total cholesterol (TC), low density lipoproteine cholesterol (LDL C), and triglycerides (TG) on the one hand, and decreased high density lipoprotein cholesterol (HDL C) on the other [2,3]. Hypertensives tend to have greater TC, LDL C, and TG resting levels, and lower resting levels of HDL C; moreover the preva lence of hyperlipidemia in hypertension is as high as 40\% [4]. Psycholog ical stress has increasingly been implied in CHD development $[5,6]$.

* Corresponding author at: Biological and Health Psychology, Department of Psychology, University of Bern, Fabrikstrasse 8, 3012 Bern, Switzerland. Tel.: +41 31631 5790; fax: + 41316314155

E-mail address: petra.wirtz@psy.unibe.ch (P.H. Wirtz).
Specifically, studies on short term physiological responses to controlled stress induction serve as a window into complex psychological and physiological processes involved in the development of CHD [7]. Recent studies also suggest that elevated physiological stress reactivity or hyper reactivity is an independent CHD risk indicator per se [8 11]. In particular, stress induced hyper activation of the sympathetic nervous system (SNS) including the cardiovascular system [8], and the hypothal amus pituitary adrenal (HPA) axis [9] have been implicated to in crease CHD risk, either by direct effects and/or by inducing adverse changes in intermediate biological risk factors including blood lipids $[10,11]$. With respect to hypertension, we recently found greater TC and LDL C changes to acute psychosocial stress in hypertensives com pared to normotensives [12]. This suggests that hypertensives are par ticularly susceptible to stress induced elevations in atherogenic lipids. Given the importance of elevated physiological stress reactivity for CHD, the observed heightened TC/LDL C stress reactivity in essential hy pertension may provide one mechanism by which stress might increase CHD risk in hypertension. 
In line with such reasoning, it might be of clinical relevance to iden tify conditions that relate to lower TC/LDL C stress reactivity in essential hypertension. We recently found higher social support and beneficial emotional regulation to be related to a reduced stress reactivity of stress hormones in hypertension [13], but studies on this issue are sparse. Pre vious research has suggested links between attributional styles and health outcomes [14 18]. Attributional styles are the habitual manner in which individuals explain positive or negative events in their lives. Seligman's theory of causal attribution distinguishes between an opti mistic and a pessimistic attributional style [19]. The optimistic attribu tional style is characterized by the tendency to explain negative events in terms of external, unstable, and situation specific causes and positive events in terms of internal, stable, and global causes [14,19]. The pessimistic attributional style is defined as the opposite of the opti mistic one. Individuals with an optimistic attributional style are regarded as more optimistic with more favorable expectancies of the fu ture than individuals with a pessimistic attributional style [14]. A variety of studies suggests that optimistic attributional styles or optimism are associated with clinically important benefits in cardiovascular outcomes $\left[\begin{array}{ll}18,20 & 22\end{array}\right]$. For example, a 10 year large scale longitudinal study dem onstrated a strong association between an optimistic attributional style and lower incidence of CHD [18]. Although the underlying pathways by which an optimistic attributional style or optimism affects cardiovascu lar outcomes are poorly understood, lower physiological stress re sponses are presumed to be involved [23]. The role of attributional styles in the interface between TC/LDL C stress reactivity and hyperten sion has not yet been studied.

The aim of this study was to investigate whether attributional styles might be associated with TC/LDL C stress reactivity in otherwise healthy, middle aged hypertensive men, compared with age matched normotensives. To induce psychosocial stress, we used the Trier Social Stress Test (TSST), a motivated performance task disguised as a job in terview [24]. This standardized stress test combines elements of uncon trollability and social evaluative threat, and creates a context in which participants are unable to get positive feedback despite best effort. Thus, the TSST can be viewed as a situation of experimentally induced failure. We assumed that the habitual manner to attribute failure to ex ternal (e.g. misfortune or task difficulty) but not to internal (e.g. effort or ability) factors might especially be associated with stress preventive ef fects because subjects would not attribute failure as self inflicted. We hypothesized that greater external stable (ES) or external variable (EV) attribution of failure and lower internal stable (IS) or internal variable (IV) attribution of failure would be associated with attenuated increases in atherogenic blood lipids (i.e. TC and LDL C) to acute stress in hypertensives relative to normotensives.

\section{Methods}

\section{Participants}

The current study is a secondary analysis of a project assessing lipid stress reactivity in essential hypertension [12] and was formally ap proved by the Ethics Committee of the State of Zurich, Switzerland. Due to known gender and menstrual cycle phase related alterations in endocrine stress reactivity [25] we recruited men only. Of a total of 45 participants, 35 men completed our attribution questionnaire. All par ticipants provided written informed consent. With the aid of the Swiss Red Cross of Zurich and through advertisements, we recruited non smoking hypertensive and normotensive men who, apart from hyper tension, were otherwise in good physical and mental health, as confirmed by an extensive health questionnaire and telephone inter view. Specific exclusion criteria, obtained by participants' self report, were as follows: regular strenuous exercise, alcohol and illicit drug abuse, any heart disease, varicosis or thrombotic diseases, elevated blood glucose level and diabetes, elevated cholesterol level, liver and renal diseases, chronic obstructive pulmonary disease, allergies and atopic diathesis, rheumatic diseases, and current infectious diseases. In addition, participants were included only if they reported no regular or occasional intake of medications. If the personal or medication histo ry was not conclusive, the participants' primary care physician was contacted for clarification.

\section{Assessment of hypertension}

After a 15 minute rest, three seated screening blood pressure (BP) measurements were obtained on three separate days by a fully auto mated sphygmomanometry device (Omron 773; Omron Healthcare Europe, Hoofddorp, The Netherlands) and the average BP was comput ed. Participants were categorized into hypertensive and normotensive individuals following the World Health Organization/International Soci ety of Hypertension definition (systolic BP $\geq 140 \mathrm{~mm} \mathrm{Hg}$ and/or diastol ic $\mathrm{BP} \geq 90 \mathrm{~mm} \mathrm{Hg}$ ) [26]. The screening procedure yielded 17 hypertensive and 18 age matched normotensive men (all with com plete attributional style data) whose characteristics are listed in Table 1. The average mean arterial pressure (MAP) across all individuals according to the formula two thirds diastolic BP + one third systolic BP was used for analysis.

\section{Psychosocial stress procedure}

All experimental sessions commenced between $2 \mathrm{pm}$ and $4 \mathrm{pm}$ and lasted for approximately $2 \mathrm{~h}$. Participants abstained from food and drinks (other than water) for $2 \mathrm{~h}$ before the experiment, and from phys ical exercise, alcohol, and caffeinated beverages starting the evening be fore the test day. To inflict psychosocial stress, we used the Trier Social Stress Test (TSST). The TSST combines a 5 minute preparation phase followed by a 5 minute mock job interview, and a 5 minute mental ar ithmetic task in front of an audience [24]. The TSST can be viewed as a situation of experimentally induced failure [27] that reliably provokes profound endocrine and cardiovascular responses $[24,28]$. During the $45 \mathrm{~min}$ before introduction to the TSST and for another $60 \mathrm{~min}$ after task completion, participants remained seated in a quiet room.

Blood for lipid and norepinephrine (NE) measures was obtained im mediately before stress, immediately after stress, and at $20 \mathrm{~min}$, and $60 \mathrm{~min}$ after stress. At the end of blood sampling, participants were debriefed and participation was remunerated with 80 Swiss francs.

\section{Measurements}

\section{External and internal attribution of failure}

External and internal attribution of failure was measured by the Ger man questionnaire for the assessment of causal attributions (IE SV F; questionnaire for the assessment of internal/external and stable/instable attributions depending on success and failure [Fragebogen zur Erfassung der internalen/externalen und stabilen/variablen Attributionen in Abhängigkeit von Erfolg und Misserfolg] [29]). Using a 5 point rating scale ranging from 1 (not at all) to 5 (absolutely), subjects were asked to rate the extent to which the statements for the given situations of fail ure applied to themselves (e.g. to be more criticized at work is just a co incidence and is independent of my work performance). The subscales external stable attribution of failure (consisting of 10 items) and external variable attribution of failure (consisting of 9 items) assess the habitual tendency to explain negative events by external stable causes (e.g. task difficulty) or external variable causes (e.g. bad luck), re spectively. The subscales internal stable attribution of failure (consisting of 10 items) and internal variable attribution (consisting of 11 items) as sess the habitual tendency to explain negative events by internal stable causes (e.g. low ability) or internal variable causes (e.g. lack of effort), respectively. Higher scores reflect a higher tendency to attribute failure to external stable, external variable, internal stable, or internal variable causes. Cronbach's alpha $(\mathrm{n}=174)$ was 0.68 (external stable), 0.69 
Table 1

Characteristics of the 35 subjects with complete attributional style assessment

\begin{tabular}{|c|c|c|c|}
\hline & $\begin{array}{l}\text { Hypertensives } \\
(n=17)\end{array}$ & $\begin{array}{l}\text { Normotensives } \\
(n=18)\end{array}$ & P-ANOVA \\
\hline Age (years) & $45.2 \pm 3.6(22.0-640)$ & $46.4 \pm 2.7(28.0-65.0)$ & .79 \\
\hline BMI $\left(\mathrm{kg} / \mathrm{m}^{2}\right)$ & $26.8 \pm 0.7(22.6-33.9)$ & $25.1 \pm 0.6(22.2-32.1)$ & .07 \\
\hline $\operatorname{MAP}(\mathrm{mm} \mathrm{Hg})$ & $111.5 \pm 1.7(97.8-121.4)$ & $92.1 \pm 1.5(82.8-104.9)$ & $<.001$ \\
\hline Cholesterol (mg/dL) & $221.8 \pm 9.8(154.0-302.0)$ & $208.7 \pm 8.9(110.0-274.0)$ & .33 \\
\hline $\mathrm{LDL}-\mathrm{C}(\mathrm{mg} / \mathrm{dL})$ & $138.7 \pm 5.6(103.0-181.0)$ & $129.3 \pm 6.7(43.0-167.0)$ & .30 \\
\hline Norepinephrine $(\mathrm{pg} / \mathrm{mL})$ & $429.0 \pm 28.0(244.3-650.7)$ & $343.6 \pm 27.1(171.4-533.2)$ & .035 \\
\hline ES & $26.4 \pm 1.4(18-38)$ & $27.7 \pm 1.3(16-38)$ & .51 \\
\hline EV & $23.7 \pm 1.4(14-36)$ & $253 \pm 1.0(19-35)$ & .37 \\
\hline IS & $232 \pm 1.3(15-39)$ & $21.9 \pm 1.0(13-28)$ & .41 \\
\hline $\mathrm{N}$ & $34.9 \pm 2.2(18-49)$ & $35.9 \pm 1.8(19-45)$ & .72 \\
\hline Neuroticism & $16.0 \pm 1.2(7-24)$ & $15.8 \pm 1.0(9-25)$ & .91 \\
\hline Extent of depression & $12.5 \pm 0.8(9-21)$ & $10.8 \pm 0.7(6-21)$ & .11 \\
\hline
\end{tabular}

Note: Values given are mean \pm SEM and range. Plasma lipid and norepinephrine measures reflect plasma levels at baseline (i.e. immediately before the stressor). BMI, body mass index; MAP, mean arterial pressure; LDL-C, low-density-lipoprotein cholesterol; ES, external-stable attribution of failure; EV, external-variable attribution of failure; IS, internal-stable attribution of failure; IV, internal-variable attribution of failure.

(external variable), 0.79 (internal stable), and 0.66 (internal variable) for the respective IE SV F subscale [29].

\section{Psychological control variables}

We assessed the extent of depression and neuroticism based on pre vious findings suggesting associations with attributional styles and/or optimism [30,31].

Extent of depression. We assessed the extent of depression by means of the short version (15 items) of the "Allgemeine Depressionsskala" (ADS K) [32]. The ADS K is the German version of the "Center of Epide miological Studies Depression Scale" (CES D) [33]. Using a 4 point rat ing scale ranging from 0 (rare) to 3 (mostly), participants were asked to rate how often they perceived a variety of depressive symptoms within the last few weeks. Higher scores mean higher extent of depres sion. Cronbach's alpha (calculated in different samples) is between 0.88 and 0.95 for the ADS K [32].

Neuroticism. Neuroticism was measured by the 6 item subscale of the shortened German version of the NEO Five Factor Inventory (NEO FFI) [34,35]. Answers were given on a 6 point rating scale ranging from 1 (strongly disagree) to 6 (strongly agree). Higher scores mean higher neuroticism. Cronbach's alpha $(n=1908)$ is 0.81 for the neuroticism subscale [35].

\section{Biochemical measures}

Blood lipids. Blood lipids (TC, HDL C, and TG) were measured in plas $\mathrm{ma}(\mathrm{mg} / \mathrm{dL})$ with a calorimetric system (AU, Olympus, Hamburg, Germany) following standard laboratory procedures (Synlab, Augs burg, Germany). LDL C was calculated using the Friedewald formu la: LDL C $=$ TC - HDL C $-(\mathrm{TG} / 2.19)$. Hemoglobin $(\mathrm{g} / \mathrm{dL})$ and hematocrit (\%) were obtained by processing whole blood collected in $2.7 \mathrm{~mL}$ EDTA tubes (Sarstedt, Rommelsdorf, Germany) on an auto mated hematology system (Advia 120, Bayer Diagnostics, Fernwald, Germany).

Norepinephrine. We previously found that NE stress reactivity predicted higher increases in TC and $\mathrm{LDL} C$ in response to acute stress [12]. There fore, we controlled for NE to account for a potential confounding influ ence on potential associations between attributional style and lipid stress reactivity. For NE assessment, venous blood was drawn into EDTA coated Monovette tubes (Sarstedt, Numbrecht, Germany) and immediately centrifuged for $10 \mathrm{~min}$ at $2000 \mathrm{~g}$. Obtained plasma was stored at $-80^{\circ} \mathrm{C}$ until analysis. Plasma NE was determined by high pressure liquid chromatography (detection limit, $0.25 \mathrm{pg} / \mathrm{mL}$; inter and intra assay coefficients of variation, $<5 \%$; Laboratory for Stress
Monitoring, Göttingen, Germany). All samples from the same subject were analyzed in the same run.

\section{Statistical analyses}

Data were analyzed using SPSS Inc. version 17.0 for Windows (Chicago, IL, USA) and presented as mean \pm SEM. All tests were two tailed with the significance level set at $p \leq .05$. $\mathrm{G}^{*}$ Power 3.1. analysis suggests that a total sample size of $N=32$ is needed to de tect an interaction effect between attributional style and plasma lipid stress reactivity in two groups with an expected medium effect size of $f=.20$ in general models with repeated measures with a power of $>.80, \alpha=.05$, given the observed minimum intercorrela tion among repeated measures of 63 and $\varepsilon=.79$. Prior to statistical analyses, data were tested for normal distribution and homogeneity of variance using Kolmogorov Smirnov and Levene's tests.

We corrected all plasma lipid levels for stress hemoconcentration following previous methods by computing stress induced changes in plasma volume (i.e., stress hemoconcentration) from hemoglobin and hematocrit measures according to the formula by Dill and Costill [36]. For plasma lipids (i.e. TC and LDL C), areas under the total response curves with respect to increase (AUCs) were calculated using the trape zoid formula [37]. NE stress changes $(\triangle N E)$ were calculated as the dif ference in NE plasma levels immediately after stress and baseline levels.

Univariate analyses of variance (ANOVAs) were calculated to test for differences in hypertensive vs. normotensive subjects with complete at tributional style assessment in terms of demographic characteristics, psychological measures, and baseline levels of plasma lipids and NE.

In order to prevent model overfitting in the subsequent main analy ses, we first analyzed data without controlling for any potential con founders. In a second step, we controlled for the cardiovascular risk factors age and body mass index (BMI) as covariates. In a third step, we additionally controlled for depression, neuroticism, and $\triangle \mathrm{NE}$ as a priori defined set of potential confounders $[31,38,39]$.

To address whether associations between attributional style and plasma lipids differ with hypertension status, we used a two step proce dure. We first (step 1) correlated attributional styles (i.e. ES, EV, IS, and IV) with baseline levels and the AUCs of plasma lipids. In order to avoid multiple testing in further analyses, we used those attributional styles that were associated with baseline levels or AUCs of plasma lipids at a level of $p<.15$. Next, we calculated moderator analyses as described by Baron and Kenny [40] (step 2). For resting plasma lipid levels, we first calculated linear regression analyses with pre stress measures of plasma lipids as dependent variables and MAP, significantly associated attributional style variables (ES, EV, IS, and/or IV, respectively), and the interaction between MAP and the respective attributional style score as independent variables. For repeated lipid measures, we 
calculated general linear models with repeated measures of plasma lipids as dependent variables. As independent variables, we again en tered MAP, significantly with AUC associated attributional style vari ables (ES, EV, IS, and/or IV, respectively), and the interaction between MAP and the respective attributional style score [13]. According to Baron and Kenny [40] statistical moderation holds if the interaction terms significantly relates to the dependent variable while controlling for the variables composing the interaction term. All variables entered in moderation analyses were $\mathrm{Z}$ transformed prior to analysis to allow calculation of interaction terms. Significant moderator effects in terms of significant associations between the respective interaction term and lipid measures were further analyzed by performing a median split on the attributional style scale rendering four subgroups of hypertensives and normotensives with either high or low values in the respective at tributional style variable (i.e. hypertensives with high and low ES, EV, IS or IV, and normotensives with high and low ES, EV, IS or IV). Subgroup differences in plasma lipid measurements were tested by ANOVAs. No tably, we used MAP as a continuous variable instead of categorizing hy pertensives and normotensives in a dichotomous variable to increase the statistical power [41].

Effect size parameters $(f)$ were calculated from partial $\eta^{2}$ values and are reported where appropriate (effect size conventions: $f: .10=$ small, $.25=$ medium, $.40=$ large) .

Results

\section{Subjects' characteristics}

Table 1 provides the characteristics of the 35 hypertensive and normotensive subjects with complete attributional style assessment. MAP and plasma levels of NE at rest were higher in hypertensives as compared to normotensives.

\section{Attributional style variables and blood lipids}

\section{Correlation analyses}

Lipid baseline levels. ES scores correlated negatively with LDL-C baseline levels $(r=.32$ $p=.065$ ). In contrast, there were no significant associations between TC or LDL-C baseline levels and IS, IV, or EV, and between TC baseline levels and ES ( $p$ 's > .23). Further controlling for age and BMI or the full set of confounders (age, BMI, neuroticism, depression, and $\triangle N E$ ) did not change the results; the associations between ES and LDL-C baseline levels actually got stronger ( $r$ 's $\left.\geq \quad .37 ; p^{\prime} s \leq .036\right)$. Moreover, ES scores correlated negatively with TC baseline levels when controlling for the full set of confounders $(r=.35, p=.059$ ).

Lipid AUCs Whereas there were no significant associations between aggregated TC or LDLC secretion and IS, IV, or EV ( $p$ 's > 25), ES scores correlated negatively with aggregated TC secretion ( $r=.28, p=.105$ ). Further controlling for age and BMI or the full set of confounders did not change the results; instead the associations between ES und aggregated TC secretion got stronger ( $r$ 's $\geq \quad .38 ; p$ 's $\leq .030)$. Moreover, ES scores correlated negatively with aggregated WL-C secretion when controlling for age and BMI $(\mathrm{c}=.27, p=.128)$ or the full set of confounders ( $r=.36, p=.054)$

\section{At rest}

There was no moderation effect of ES with hypertension status in pre-stress plasma lipid levels ( $p$ 's $>.52$ ).

\section{Stress reactivity}

Moderation testing revealed a significant MAP-by-ES interaction for TC stress reactivity $\left(F(2.9 / 90.1)=3.71, p=.015, \eta_{p}^{2}=.107, f=.35 ; F i g .1\right)$ and LDL-C stress reactivity $(F(3.0)$ $93.0)=3.61, p=.016, \eta_{p}^{2}=.104, f=34$, Fig. 2). Post-hoc tests showed that TC levels were highest in hypertensives with low ES immediately after stress ( $p$ 's $\leq .020$ ). In contrast, hypertensives with high ES did not differ significantly from normotensives in TC immediately after stress ( $p$ 's > .68). Similarly, in terms of LDL-C, hypertensives with low ES showed highest LDL-C secretion immediately after stress ( $p$ 's $\leq .039$ ). Moreover, hypertensives with high ES did not significantly differ from normotensives in LDL-C immediately after stress ( $p$ 's $>28$ ). Further controlling for age and BMI (interaction MAP-by-ES and TC stress reactivity: $F(3.0 / 87.0)=3.43, p=.022, \eta_{\mathrm{p}}^{2}=.106, f=.34$; interaction MAP-by-ES andLLC stress reactivity: $F(3.0 / 87.0)=3.34, p=.023, \eta_{p}^{2}=.103, f=34$ ) or the full set of confounders (interaction MAP-by-ES and TC stress reactivity: $F(3.0 / 78.0)=4.27, p=.008$, $\eta_{p}^{2}=.141, f=A 1$; interaction MAP-by-ES and LDL-C stress reactivity: $F(3.0 / 78.0)=4.05$, $p=.010,12=.135, f=40$ ) did not significantly change results.

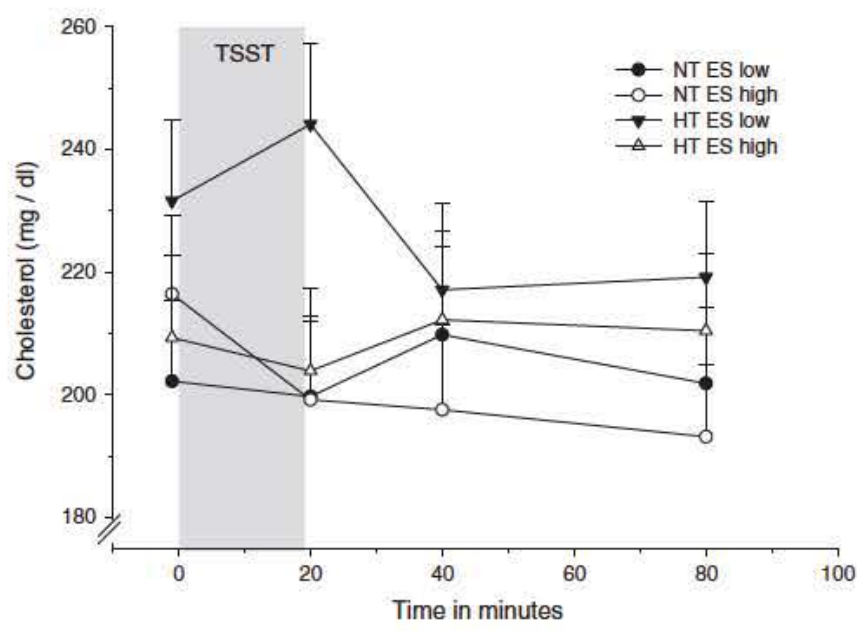

Fig. 1. Total cholesterol reactivity to psychosocial stress in hypertensive (HT) and normotensive (NT) men with high and low external-stable attribution to failure (ES). Values are means and simple error bars for SEM.

\section{Conclusion}

This is the first study to investigate associations between attribu tional styles and blood lipid changes to acute psychosocial stress in hy pertensive men, compared with age matched normotensives. The main finding of our study was that in hypertensive men, but not in normoten sives, lower ES scores were associated with higher TC and LDL C chang es to psychosocial stress. Moreover, hypertensives with higher ES scores did not differ significantly from normotensives in their TC and LDL C stress changes. These associations showed large effect sizes and were also independent of a broad set of potential confounders including car diovascular risk factors, NE stress change, and related psychological constructs. In contrast, there were no associations between blood lipids at rest and ES, or blood lipids (at rest as well as aggregated secretion) and EV, IS, and IV.

Our data suggest that the habitual manner of hypertensive subjects to attribute failure to external stable causes, such as task difficulty, re lates to the extent to which atherogenic lipid levels increase following stressful events: the higher the ES, the lower the stress induced TC and LDL C increases in individuals with hypertension. Several decades

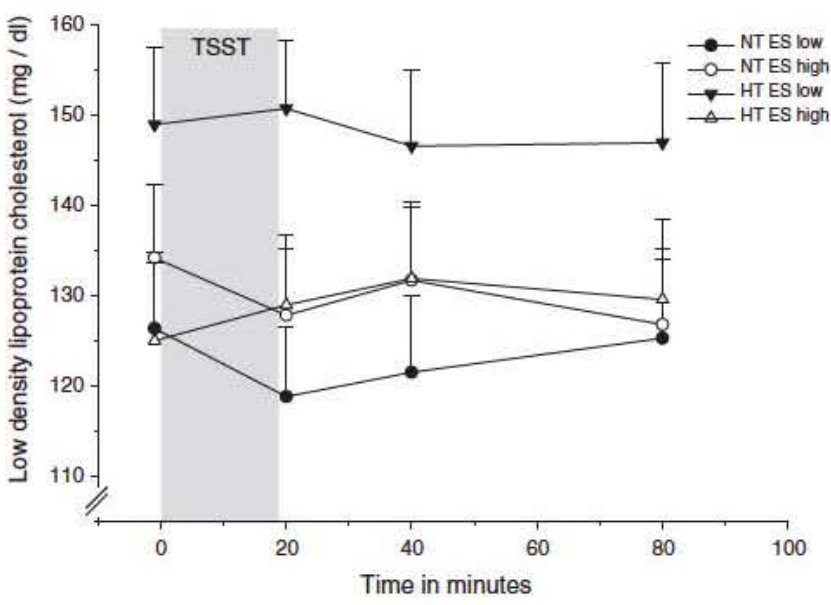

Fig. 2. Low-density-lipoprotein cholesterol reactivity to psychosocial stress in hypertensive (HT) and normotensive (NT) men with high and low external-stable attribution to failure (ES). Values are means and simple error bars for SEM. 
of research have provided strong empirical evidence that elevated TC and LDL C blood levels substantially increase the risk of atherosclerotic cardiovascular disease, especially among hypertensive patients $[2,3,42]$. However, it is unknown whether, and if so, to what extent acutely in creased atherogenic lipids deposit at sites of endothelial lesions, thereby contributing to the initiation of fatty streaks, atherosclerosis progres sion, and ultimately coronary occlusion. Consequently, the clinical rele vance of any dampening effect ES may have on stress reactivity of atherogenic lipids in hypertensive subjects remains to be elucidated. Notwithstanding this, it has been shown that acute lipid stress reactivity predicts higher LDL $\mathrm{C}$ levels and TC/HDL C ratios several years later [43]. Thus, over a lifespan, an acutely exaggerated lipid response might contribute to clinically manifest atherosclerosis in hypertensives. Therefore, ES might be a protective factor for stress induced lipid relat ed atherogenesis in essential hypertension. However, further studies are needed to determine the implications of our observations in health, essential hypertension, and CHD.

To the best of our knowledge, no study has yet investigated the ef fects of attributional styles on lipid responses to acute stress, neither in healthy individuals nor in hypertensive subjects. Our study provides first indications of a possible relationship between ES, stress, and ath erogenesis in essential hypertension. The findings described here are in line with research on beneficial effects of optimistic attributional styles or optimism, respectively, on cardiovascular outcomes. For in stance, a prospective study found that optimism predicts a lower rate of and longer period before rehospitalization after coronary bypass sur gery [44]; moreover, a 3 year longitudinal study found that optimists are less likely to show increases in carotid intima medial thickness (a subclinical marker of atherosclerosis) than pessimists [21]; a recent meta analytic review also demonstrated that heightened levels of opti mism are associated with reduced risk of CHD [20]. Individuals who usually explain negative events by causes that are external, unstable, and specific ("it's bad luck but not me"), are said to have an optimistic attributional style; thus, external attribution of failure can be interpreted as an optimistic attributional style.

EV can also be interpreted as an optimistic explanatory style making the dampening effect on lipid stress changes only for ES, but not for EV puzzling. However, our sample size was relatively small and the vari ance within the EV variable was also smaller than in the ES variable. We therefore cannot rule out methodological causes for the non significant EV effects. The strong positive association between EV and ES in our sample (data not shown) further corroborates this notion. We thus feel the non significant EV effect should be interpreted with caution.

Interestingly, we also failed to find any association between the ha bitual manner of hypertensive subjects to attribute failure to internal stable causes (such as e.g. ability), or internal variable causes (such as e.g. effort) and stress induced increases in atherogenic lipid levels. Given that IS and IV can be interpreted as a pessimistic attributional style $[14,19]$ our findings suggest effects on atherogenic blood lipid changes to acute psychosocial stress in hypertensive subjects only for optimistic but not for pessimistic explanatory styles. Notably, this is in line with studies suggesting that optimism and pessimism are distinct constructs $[45,46]$.

What are the underlying pathways by which ES impacts lipid stress changes in hypertensives, but not in normotensives? Lipid stress reac tivity is thought to be caused by multiple mechanisms, including stress associated alterations in plasma volume and alterations in lipid metabolism during stress [ 47 50]. It is unlikely that the observed effects of ES on lipid stress changes are a concomitant phenomenon of stress hemoconcentration, as we controlled for stress induced shifts in hemoconcentration before all analyses. We can only speculate that un derlying mechanisms may include altered lipid metabolism. We further speculate that an increased tendency to attribute negative events or fail ure to external stable causes (such as task difficulty) is associated with a reduced perception of stress and, as a result, with a reduction in neuroendocrine stress responses, which in turn relate to lipid stress re activity [12]. To examine if NE moderates the association between ES and lipid changes in response to stress, we statistically controlled for NE stress changes. However, our findings even became stronger after controlling for NE stress changes, suggesting that although NE stress changes independently related to higher lipid stress responses [12] they did not confound lipid changes to stress in relation to ES. Future studies are needed to examine additional pathways that may mediate ES related lipid stress changes. For example it remains to be tested whether ES relates to alterations in cognitive processes such as stress appraisal or state anxiety that in turn may relate to alterations in endo crine stress reactivity that may affect lipid stress reactivity.

What are the potential therapeutic implications of our study? Since we found in our cross sectional data that hypertensives with higher ES show lower lipid stress reactivity we speculate that it is more likely that ES is a cause rather than a consequence of the lower lipid stress re activity. Given this, therapeutic interventions in hypertensives may aim at increasing ES e.g. by cognitive restructuring. However, it remains to be studied whether a potential intervention induced increase in ES con sequently reduces lipid stress reactivity in hypertensives.

Our study has several strengths, which include recruitment of appar ently healthy and unmedicated subjects with reasonable health habits. This is crucial since blood lipid metabolism is affected by several drugs, including antihypertensives, and lifestyle habits [51]. We also controlled for a variety of known and potential confounders to rule out a potential confounding influence on the measured variables. More over, we corrected plasma lipid levels for stress induced shifts in hemoconcentration prior to all analyses. Finally, we used a highly stan dardized and potent stress test that reliably induces neuroendocrine stress responses. The study also has its limitations. First, its cross sectional nature does not allow us to interpret the direction of the ES lipid stress link in hypertensives. However, as our attributional style measure refers to a habitual manner and as we induced lipid stress re activity acutely, our study design may support the conclusion that it is ES that likely attenuates lipid stress reactivity rather than vice versa. Second, our sample size was relatively small and included only appar ently healthy men. Thus, our findings may not be generalized to women and hypertensive patients with overt CHD. Moreover, it remains to be tested whether our findings also apply to hypertensive patients on antihypertensive treatment, particularly if the patient presents with ES. Given that we found NE stress increases to relate to lipid stress reactiv ity [12] one could speculate that antihypertensive treatment particular ly with adrenergic blockers may prevent or reduce stress induced catecholamine increases and consequently catecholamine induced ef fects such as potential lipid increases. Future studies are needed to de termine the implications of our observations in hypertensive patients on antihypertensive treatment.

Taken together, our data suggest a buffering effect of ES on athero genic blood lipid changes to acute psychosocial stress in men with es sential hypertension. Our observations require replication in larger samples, possibly in naturalistic settings, and other populations includ ing patients on antihypertensive treatment. Moreover, the clinical im plications of our observations in health, essential hypertension, and CHD need to be demonstrated.

\section{Conflict of interest statement}

The authors have no competing interests to report.

\section{Acknowledgments}

This study was funded by the Swiss National Science Foundation grant PP00P1_128565/1 and grant 2003 from the University of Zurich, Switzerland (both to PHW). 


\section{References}

[1] Kaplan NM. Importance of coronary heart disease risk factors in the management of hypertension. An overview. Am J Med 1989;86:1-4

[2] Baigent C, Keech A, Kearney PM, Blackwell L, Buck G, Pollicino C, et al. Efficacy and safety of cholesterol-lowering treatment: prospective meta-analysis of data from 90,056 participants in 14 randomised trials of statins. Lancet 2005;366:1267-78.

[3] Castelli WP. Lipids, risk factors and ischaemic heart disease. Atherosclerosis 1996 S1-9.

[4] Bonaa KH, Thelle DS. Association between blood pressure and serum lipids in a population. The Tromso Study. Circulation 1991;83:1305-14.

[5] Brotman DJ, Golden SH, Wittstein IS. The cardiovascular toll of stress. Lancet 2007;370:1089-100.

[6] Steptoe A, Kivimaki M. Stress and cardiovascular disease. Nat Rev Cardiol 2012:9:360-70

[7] Linden W, Gerin W, Davidson K. Cardiovascular reactivity: status quo and a research agenda for the new millennium. Psychosom Med 2003;65:5-8.

[8] Chida Y, Steptoe A. Greater cardiovascular responses to laboratory mental stress are associated with poor subsequent cardiovascular risk status: a meta-analysis of prospective evidence. Hypertension 2010;55:1026-32.

[9] Hamer M, Endrighi R, Venuraju SM, Lahiri A, Steptoe A. Cortisol responses to menta stress and the progression of coronary artery calcification in healthy men and women. PLoS One 2012; 7:e31356.

[10] Niaura R, Stoney CM, Herbert PN. Lipids in psychological research: the last decade. Biol Psychol 1992;34:1-43.

[11] Kop WJ. Chronic and acute psychological risk factors for clinical manifestations of coronary artery disease. Psychosom Med 1999;61:476-87.

[12] Wirtz PH, Ehlert U, Bartschi C, Redwine LS, von Kanel R. Changes in plasma lipids with psychosocial stress are related to hypertension status and the norepinephrine stress response. Metabolism 2009;58:30-7.

[13] Wirtz PH, von Kanel R, Mohiyeddini C, Emini L, Ruedisueli K, Groessbauer S, et al. Low social support and poor emotional regulation are associated with increased stress hormone reactivity to mental stress in systemic hypertension. J Clin Endocrinol Metab 2006;91:3857-65.

[14] Lin EH, Peterson C. Pessimistic explanatory style and response to illness. Behav Res Ther 1990;28:243-8.

[15] Singh JA, O'Byrne MM, Colligan RC, Lewallen DG. Pessimistic explanatory style: a psychological risk factor for poor pain and functional outcomes two years after knee replacement. J Bone Joint Surg 2010;92:799-806.

[16] Jackson B, Sellers RM, Peterson C. Pessimistic explanatory style moderates the effect of stress on physical illness. Pers Individ Differ 2002:32:567-73.

[17] Maruta T, Colligan RC, Malinchoc M, Offord KP. Optimism-pessimism assessed in the 1960s and self-reported health status 30 years later. Mayo Clinic Proc 2002;77:748-53.

[18] Kubzansky LD, Sparrow D, Vokonas P, Kawachi I. Is the glass half empty or half full? A prospective study of optimism and coronary heart disease in the normative aging study. Psychosom Med 2001:63:910-6.

[19] Seligman M. Learned Optimism. New York: Pocket Books; 1998.

[20] Rasmussen HN, Scheier MF, Greenhouse JB. Optimism and physical health: a metaanalytic review. Ann Behav Med 2009;37:239-56.

[21] Matthews KA, Raikkonen K, Sutton-Tyrrell K, Kuller LH. Optimistic attitudes protect against progression of carotid atherosclerosis in healthy middle-aged women. Psychosom Med 2004:66:640-4.

[22] Tindle HA, Chang YF, Kuller LH, Manson JE, Robinson JG, Rosal MC, et al. Optimism, cynical hostility, and incident coronary heart disease and mortality in the Women's Health Initiative. Circulation 2009;120:656-62.

[23] Carver CS, Scheier MF, Segerstrom SC. Optimism. Clin Psychol Rev 2010;30:879-89.

[24] Kirschbaum C, Pirke KM, Hellhammer DH. The 'Trier Social Stress Test'-a tool for investigating psychobiological stress responses in a laboratory setting. Neuropsychobiology 1993;28:76-81.

[25] Kirschbaum C, Kudielka BM, Gaab J, Schommer NC, Hellhammer DH. Impact of gender, menstrual cycle phase, and oral contraceptives on the activity of the hypothalamus-pituitary-adrenal axis. Psychosom Med 1999;61:154-62.

[26] Kjeldsen SE, Erdine S, Farsang C, Sleight P, Mancia G, Subcommittee WIHG. 1999 WHO/ISH Hypertension Guidelines-highlights \& ESH update. J Hyperten 2002;20:153-5.
[27] Dickerson SS, Mycek PJ, Zaldivar F. Negative social evaluation, but not mere social presence, elicits cortisol responses to a laboratory stressor task. Health Psychol 2008;27:116-21.

[28] Dickerson SS, Kemeny ME. Acute stressors and cortisol responses: a theoretical integration and synthesis of laboratory research. Psychol Bull 2004;130:355-91.

[29] Dorrman W, Hinsch R. Der IE-SV-F: Ein differentieller Fragebogen zur Erfassung von Attributionsgewohnheiten in Erfolgs- und Misserfolgssituationen. Diagnostica 1981;27:360-78.

[30] Kennedy DK, Hughes BM. The optimism-neuroticism question: an evaluation based on cardiovascular reactivity in female college students. Psychol Rec 2004;54:373-86.

[31] Jowsey SG, Cutshall SM, Colligan RC, Stevens SR, Kremers WK, Vasquez AR, et al. Seligman's theory of attributional style: optimism, pessimism, and quality of life after heart transplant. Prog Transplant 2012;22:49-55.

[32] Hautzinger M, Bailer M. Allgemeine Depressionsskala. Weinheim: Beltz Test GmbH; 1993.

[33] Radloff LS. The CES-D scale: a self-report depression scale for research in the general population. Appl Psychol Meas 1977;3:385-401.

[34] Ostendorf F. Sprache und Persönlichkeitsstruktur. Zur Validität des Fünf-FaktorenModells der Persönlichkeit. Regensburg: Roderer; 1990.

[35] Korner A, Geyer M, Roth M, Drapeau M, Schmutzer G, Albani C, et al. Personality assessment with the NEO-Five-Factor Inventory: the 30-Item-Short-Version (NEO-FFI30. Psychother Psychosom Med Psychol 2008;58:238-45.

[36] Dill DB, Costill DL. Calculation of percentage changes in volumes of blood, plasma, and red cells in dehydration. J Appl Physiol 1974;37:247-8.

[37] Pruessner JC, Kirschbaum C, Meinlschmid G, Hellhammer DH. Two formulas for computation of the area under the curve represent measures of total hormone concentration versus time-dependent change. Psychoneuroendocrinology 2003;28:916-31.

[38] Wirtz PH, Redwine LS, Ehlert U, von Kanel R. Independent association between lower level of social support and higher coagulation activity before and after acute psychosocial stress. Psychosom Med 2009;71:30-7.

[39] Alloy LB, Peterson C, Abramson LY, Seligman ME. Attributional style and the generality of learned helplessness. J Pers Soc Psychol 1984;46:681-7.

[40] Baron RM, Kenny DA. The moderator-mediator variable distinction in social psychological research: conceptual, strategic, and statistical considerations. J Pers Soc Psychol 1986;51:1173-82.

[41] MacCallum RC, Zhang S, Preacher KJ, Rucker DD. On the practice of dichotomization of quantitative variables. Psychol Methods 2002;7:19-40.

[42] Padwal R, Straus SE, McAlister FA. Evidence based management of hypertension. Cardiovascular risk factors and their effects on the decision to treat hypertension: evidence based review. BM] 2001;322:977-80

[43] Steptoe A, Brydon L. Associations between acute lipid stress responses and fasting lipid levels 3 years later. Health Psychol 2005;24:601-7.

[44] Scheier MF, Matthews KA, Owens JF, Schulz R, Bridges MW, Magovern GJ, et al. Optimism and rehospitalization after coronary artery bypass graft surgery. Arch Intern Med 1999;159:829-35.

[45] Marshall GN, Wortman CB, Kusulas JW, Hervig LK, Vickers RR. Distinguishing optimism from pessimism: relations to fundamental dimensions of mood and personality. J Pers Soc Psychol 1992:1067-74.

[46] Robinson-Whelen S, Kim C, MacCallum RC, Kiecolt-Glaser JK. Distinguishing optimism from pessimism in older adults: is it more important to be optimistic or not to be pessimistic? J Pers Soc Psychol 1997;73:1345-53.

[47] Muldoon MF, Bachen EA, Manuck SB, Waldstein SR, Bricker PL, Bennett JA. Acute cholesterol responses to mental stress and change in posture. Arch Intern Med 1992; 152:775-80.

[48] Stoney CM, West SG, Hughes JW, Lentino LM, Finney ML, Falko J, et al. Acute psychological stress reduces plasma triglyceride clearance. Psychophysiology 2002;39:80-5.

[49] Bacon SL, Ring C, Lip GY, Carroll D. Increases in lipids and immune cells in response to exercise and mental stress in patients with suspected coronary artery disease: effects of adjustment for shifts in plasma volume. Biol Psychol 2004;65:237-50.

[50] Stoney CM, Niaura R, Bausserman L. Temporal stability of lipid responses to acute psychological stress in middle-aged men. Psychophysiology 1997;34:285-91.

[51] Ansell B. Future directions in lipid therapies. Adv Ther 2002;19:61-72. 